\title{
LOGIC AND INVARIANT THEORY. I: INVARIANT THEORY OF PROJECTIVE PROPERTIES
}

BY

\author{
WALTER WHITELEY (1)
}

ABSTRACT. This paper initiates a series of papers which will reexamine some problems and results of classical invariant theory, within the framework of modern first-order logic. In this paper the notion that an equation is of invariant significance for the general linear group is extended in two directions. It is extended to define invariance of an arbitrary first-order formula for a category of linear transformations between vector spaces of dimension $n$. These invariant formulas are characterized by equivalence to formulas of a particular syntactic form: homogeneous formulas in determinantsi or "brackets". The fuller category of all semilinear transformations is also introduced in order to cover all changes of coordinates in a projective space. Invariance for this category is investigated.

The results are extended to cover invariant formulas with both covariant and contravariant vectors.

Finally, Klein's Erlanger Program is reexamined in the light of the extended notion of invariance as well as some possible geometric categories.

Introduction. Classical invariant theory, after a series of ups and downs, has recently enjoyed new vogue, first with the well-known book of Hermann Weyl [10] and more recently with the extensive survey article by Dieudonné and Carrell [2]. In this series of articles we will employ the tools of the first-order predicate calculus, both model theory and proof theory, to recast and extend some of the basic results of the invariant theory.

Invariant theory considered polynomials and polynomial equations in the coordinates of points in a projective space and asked which such expressions are "invariant" under changes of coordinates for the space? It was noticed quite

Received by the editors December 13, 1971.

AMS (MOS) subject classifications (1970). Primary 02H15, 15A72, 18F99, 50A20, 50D99; Secondary 02H05, 13L05, 14A25, 20G15, 50A15, 50D30.

Key words and phrases. Analytic projective geometry, category of models, invariance for a category, invariant first-order formula, semilinear transformation, projective properties, vector space, change of coordinates, general linear group, determinant, bracket, Klein's Erlanger Program.

(1) Portions of this work are taken from the author's Ph.D. thesis written at the Massachusetts Institute of Technology under the supervision of Professor G.-C. Rota. Preparation for publication was supported in part by NRC grant A8226. 
early that a fundamental role was played by the so-called "brackets", namely determinants of subsets of $n$ points, where $n-1$ is the dimension of the projective space. The First Fundamental Theorem of Invariant Theory states that polynomials that are relative invariants are expressible as homogeneous polynomials in the brackets $[10$, p. 45$],[8$, p. 312]. Gram's theorem characterized invariant polynomial equations in terms of conjunctions of homogeneous equations in the brackets [8, p. 271].

Our purpose in this paper is to describe general first-order formulas which are invariant under changes of coodinate system. In view of developments both in logic and in geometry, we believe these are natural questions to ask. Many properties and theorems of classical synthetic geometry are expressed by means of logical connectives and quantifiers, and thereby require full first-order logic for their expression. Our characterization is a natural extertsion of Gram's theorem, stating that invariant formulas are homogeneous formulas in the determinants. We present the characterization in two stages, first for open or quantifierfree formulas and then for general formulas.

The development has a natural logical setting which provides a unified view on all the classical invariant theories as well as some theorems of mathematical logic. This notion of "invariance for a'category of models" allows us to recognize some other natural questions of invariance, and to extend Klein's notion that a geometry is given as the invariant theory of a group of transformations [6, p. 130].

In future papers we will examine homogeneous coordinates [13], axiom systems for analytic geometry in the invariants and their relationship to the Second Fundamental Theorem of Invariant Theory, and applications to combinatorial geometric questions and representable matroids.

We have found that the combination of these results with some techniques in the proof theory of integral domains explains the observed standard form of a wide class of theorems of projective geometry: identities in the ring of brackets. The analysis also yields a practical procedure for finding the identities corresponding to the theorems [11]. The presentation of theorems as identities and conjunctions of identities is the form best suited to investigating translations and generalizations to other settings such as modular lattices or combinatorial geometry. It is also the form anticipated by earlier workers [4, Preface].

1. Languages and definitions. We must begin by defining the first-order languages suitable for the analytic theory of projective geometry, where the field extends a given fixed field $K$. By convention, if $K=\varnothing$ we are referring to geometries over an arbitrary field or all analytic models of Pappian synthetic projective geometry. 
Definition 1.l. $L n(S A K)$, the simplified language for analytic geometrics of $\operatorname{dim} n-1$ extending a field $K$, is the modified two-sorted language of type

$$
\tau=\left\{V, S ;=; e^{1}, e^{2}, \cdots, e^{n},+, \cdot,-; 0,1,\left\langle c_{f}\right\rangle_{f \in K}\right\}
$$

and signature

$\sigma:=$ is a relation between terms of sort $S$,

$e^{1}, \cdots, e^{n}$ are operations $V \rightarrow S$,

+ and $\cdot$ are operations $S \times S \rightarrow S$,

- is an operation $S \rightarrow S$,

0,1 , and $c_{f}, f \in K$, are constants of sort $S$,

and only variables of sort $V$ are allowed.

Definition 1.2. A standard model of $\operatorname{Ln}(S A K)$ is a model

$$
U=\left\{V, S ;=; e^{1}, \cdots, e^{n},+, \cdot,-; 0,1,\langle b(f)\rangle_{f \in K}\right\}
$$

where $S$ is a field, $V$ is a vector space of $\operatorname{dim} n$ over $S$ and = is equality in the field $S$. The operations and constants are as follows:

$e^{1}, \cdots, e^{n}$ are the projections of a vector onto its components

$$
e^{i}\left(v_{1}, \cdots, v_{n}\right)=v_{i},
$$

,$+ \cdot$, and - are the domain operations on the field $S$,

0,1 are the zero and unit of the field $K$,

$b$ is an isomorphism of $K$ into the field $S$, if $K \neq \varnothing$.

The collection of all standard models of $L n(S A K)$ is denoted $V n K$, vector spaces of $\operatorname{dim} n$ over fields extending $K$.

Remarks. Two comments are worth making about this choice of language.

One concerns the omission of the vector space operations + and - and the scalar multiplication $*$, as well as the field operation ${ }^{-1}$. We could easily construct languages $L n(A K)$ and models $U n K$ containing these operations. However, for the standard models we find that for any formula $F$ in $\operatorname{Ln}(A K)$ there is a formula $S\left(F_{1}\right)$ in $L n(S A K)$ such that $F \leftrightarrow S(F)$ in all standard models UnK. That is, $F$ and $S(F)$ define the same property in each of the standard models. To eliminate,+- and $*$ for the vectors we repeatedly use the fact that the $e^{i}$ are linear in all standard models: $e^{i}\left(a * v+v^{\prime}\right)=a \cdot e^{i}(v)+e^{i}\left(v^{\prime}\right)$. Since these identities hold in all $U n K$, we carry out these substitutions in $F$ creating $F^{\prime}$. Only terms of sort $S$ occur in the relation =, so this eliminates all occurences. After making ${ }^{-1}$ an operation in all the fields, say by defining $0^{-1}=0$, we can eliminate ${ }^{-1}$ from all equations in $F$, by taking two cases: $t=0$, where we substitute 0 for $t^{-1}$, and $t \neq 0$, where we multiply through to clear fractions. The net result is the formula $S(F)$ in $\operatorname{Ln}(S A K)$ which was promised.

The second omission is the variables for elements of the field $S$. It is a 
well-observed phenomenon of analytic projective geometry and linear algebra that all reasonable conditions can be expressed without resorting to variables for the field elements. There are two ways to view this. One is the simple reason that, given a variable over the field; say $k$, we can replace it by a variable $x$ over the vector space and replace occurences of $k$ with $e^{1}(x)$. The deeper reason will be explained in the fourth paper in this series when we recognize that the properties traditionally studied are "combinatorial" and we can eliminate all quantifiers.

In classical invariant theory vector spaces were viewed as classes of homogeneous coordinates of some projective space and interest centered on invariant properties or properties not affected by maps of the vector space which corresponded to changes of coordinate system for the space. These maps are the collineations which map lines onto lines in the projective sense.

The First Fundamental Theorem of Projective Geometry characterizes collineation maps, for spaces of $\operatorname{dim} 3$ or more, as semilinear transformations or transformations which are composites of linear transformations $T$, given by nonsingular matrices $[T]$, and automorphisms $A$, induced by taking an automorphism of the field and applying this to all coordinates [1, p. 44]. Classical workers were principally concerned with a projective geometry over the reals and therefore concentrated on the general linear group, $G l(n)$, over the reals.

An appropriate setting for these questions of invariance is in terms of particular categories of models of projective geometry and their collineations. We will define a series of three categories to be considered in this paper. Particular cases of the first two were considered in the classical theory.

Definition 1.3. $S l(n K)$ is the category with objects $V n K$ and morphisms given by matrices $[T]$ with determinant 1 . For a vector $v$ written as an $n \times 1$ matrix $[v], T(v)$ is $[T] \times[v]$.

Definition 1.4. $G l(n K)$ is the category with objects $V n K$ and morphisms given by nonsingular matrices $[T]$.

Definition 1.5. $\mathrm{Pa}(n K)$ is the category with objects $V n K$ and morphism arbitrary compositions of the maps of $G l(n K)$ and maps $T a:\left(v_{1}, \cdots, v_{n}\right) \rightarrow$ $\left(a\left(v_{1}\right), \cdots, a\left(v_{n}\right)\right)$ where $a$ is a field isomorphism of the field underlying the particular vector space which is the domain of $\mathrm{Ta}$.

Notice that the morphisms are not necessarily structural isomorphisms of the models in the category.

The notion of invariance can now be made specific by rewording the classical notion of the invariant significance of an equation.

Definition 1.6. A formula $F$ is invariant for a category of models $C$ iff for every morphism $T$ in $C$ with domain $M, M$ satisfies $F\left(m_{1}, \cdots, m_{p}\right)$ iff $T(M)$ satisfies $F\left(T\left(m_{1}\right), \cdots, T\left(m_{p}\right)\right)$. 
Our task is to characterize syntactically, as fully as possible, properties invariant for $P a(n K)$. In the process we will consider formulas invariant for each of the categories defined above.

Remarks. If the category $C$ contains only isomorphisms of models, every formula in the language is invariant for $C$. This is the basis for work on logic as invariant theory [7]. If the category is the collection of all models of a theory together with all structural embeddings, invariance for the category coincides with the notion of invariance for extension. When the theory is a universal theory, a theorem of logic characterizes formulas invariant for such a category as those formulas equivalent, within the theory, to quantifier-free formulas [3, p. 75].

For a general category of models we could mimic the practice in logic and consider: a formula is persistent for a category $C$ iff for $T$ a morphism with domain $M, M \vDash F\left(m_{1}, \cdots, m_{n}\right)$ implies $T(M) \vDash F\left(T\left(m_{1}\right), \cdots, T\left(m_{n}\right)\right)$. If the category is such that each morphism has an inverse, such as $G l(n K)$ or $P a(n K)$, then persistent for $C$ and invariant for $C$ are synonymous. In general, $F$ is invariant for $C$ iff $F$ and $\urcorner F$ are persistent for $C$.

From classical invariant theory, specifically Gram's theorem and the First Fundamental Theorem of Invariant Theory, we have a strong hunch about the syntactic form of invariant formulas. For equations invariant for $S l(n)$ or $G l(n)$ we are told they can be forced to be formulas where the basic terms involving vectors are determinants or "brackets".

We now present a language for such formulas. For simplicity, because our concern is with the significance of formulas, we will omit the operators +, -, and * on vectors as well as $\div$ on scalars, although these operations will appear in the basic language for proving theorems which will be presented in Logic and invariant theory. III.

Definition 1.7. The language $L n(S B K)$, the simplified language of brackets, is a modified two-sorted language of type

and signature

$$
\tau=\left\{V, S ;=;[],+, \cdot,-; 0,1,\left\langle c_{f}\right\rangle_{f \in K}\right\}
$$

$\sigma:=$ is a relation between terms of sort $S$ only,

[] is an operation $V^{n} \rightarrow S$,

+ and $\cdot$ are operations $S \times S \rightarrow S$,

- is an operation $S \rightarrow S$,

0,1 , and $c_{f}, f \in K$, are constants of sort $S$, and only variables of sort $V$ occur.

Definition 1.8. A standard model of $L n(S B K)$ is a model

$$
W=\left\{V, S ;=; \operatorname{det},+, \cdot,-; 0,1,\langle b(f)\rangle_{f \in K}\right\}
$$


where $V$ is a vector space of dimension $n$ over the field $S$ and $=$ is the equality relation on $S$. The operations and constants are as follows: det is the operation $V^{n} \rightarrow S$ which carries $\left(v_{1}, \cdots, v_{n}\right)$ into the determinant of the matrix $\left[v_{1} \cdots v_{n}\right]$ with the vectors written as columns of the matrix,

,$+ \cdot$, and - are the usual operations on $S$,

0 and 1 are the zero and unit of $S$,

$b$ is a field injection of $K$ into $S$.

The collection of all standard models is denoted $B n K$.

In this language the atomic formulas are equations $s=t$ where $s$ and $t$ can be viewed as polynomials in brackets $\left[v_{1} \cdots v_{n}\right]$ with coefficients in $K$. For easier description we give the following definitions.

Definition 1.9. A formula $F$ in $L n(S B K)$ is homogeneous iff each atomic formula appearing in $F$ is an equation homogeneous in occurences of the brackets.

Definition 1.10. Det: $V n K \rightarrow B n K$ is the function such that $\operatorname{Det}(U)$ is the model with the same underlying vector space, field, and injection of $K$, with $\operatorname{det}\left(v_{1} \cdots v_{n}\right)$ interpreted as the determinant of the matrix $\left[e^{i}\left(v_{j}\right)\right]$ in $U$. This function is a surjection onto $B n K$.

Definition 1.11. Det: $L n(S B K) \rightarrow L n(S A K)$ is the embedding of formulas obtained by extending the basic map: $\left[v_{1} \cdots v_{n}\right] \rightarrow \operatorname{det}\left[e^{i}\left(v_{j}\right)\right]$.

The connection between these maps is illustrated by the basic fact that $\operatorname{Det}(U) \vDash F\left(v_{1}, \cdots, v_{m}\right)$ iff $U \vDash \operatorname{Det}\left(F\left(v_{1}, \cdots, v_{m}\right)\right)$.

2. Invariant open formulas. The following result is a translation into our framework of the classical theorem of Gram.

An equation $E$ in $L n(S A K)$ is invariant for $G l(n K)$ iff there are homogeneous equations $G_{1}, \cdots, G_{m}$ in $L n(S B K)$, with the same free variables plus perhaps $n$ new variables $c_{1}, \cdots, c_{n}$ such that $E \leftrightarrow \operatorname{Det}\left(\forall c_{1} \cdots \forall c_{n}\left(G_{1} \& \cdots \& G_{m}\right)\right)$ in all models in $V n K$.

The classical theory considered only fields $K$ of characteristic 0 but the techniques can be simplified to apply equally well to other fields $K$ and to the case $K=\varnothing$. In this section we will extend this theorem to open formulas and invariance for $S l(n K), G l(n K)$ and $P a(n K)$.

In the next section we will characterize quantified formulas invariant for these categories. However, the methods used will not, in general, provide the same amount of information for quantifier-free formulas. The methods employed here will increase our information about the structure of open, invariant formulas in a practical sense. It seems of sufficient interest to extract this information and an algorithm for open formulas and then move on to the more general case. 
Theorem 2.1. An open formula $F$ in $L n(S A K)$ is invariant for $S l(n K)$ iff there is an open formula $G$ in $L n(S B K)$, with the same free variables plus $c_{1}$, $\cdots, c_{n}$, sucb that $F \leftrightarrow \operatorname{Det}\left(\left(\forall c_{1}, \cdots, c_{n}\right) G\right)$ in the theory of $V n K$.

Proof. Part I. Assume $F$ is invariant for $S l(n K)$. We will construct the required $G$.

Take any model $V$ in $V n K$ and any vectors $c_{1}, \cdots, c_{n}$ in $V$. From these construct the matrix $\left[c_{1} \cdots c_{n}\right]$ and the transformation $T$ with matrix $\left[C_{i j}\right]$ where $C_{i j}$ is the cofactor of $c_{j i}$ in the matrix $\left[c_{1} \cdots c_{n}\right]$.

If $\operatorname{det}\left[c_{1} \cdots c_{n}\right]=1$ then $\operatorname{det}[T]=1$ and $T$ is a morphism in $\operatorname{Sl}(n K)$. With this assumption

$$
V \vDash F\left(v_{1}, \cdots, v_{m}\right) \quad \text { iff } \quad T(V) \vDash F\left(T\left(v_{1}\right), \cdots, T\left(v_{m}\right)\right) .
$$

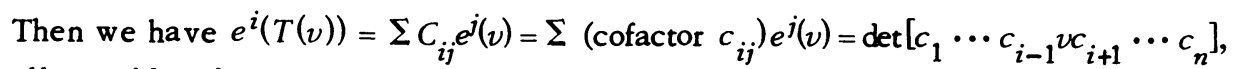
all considered as equations in the field $S$ of the model $V$. Also, $T(V)$ has the same field as $V$ so

$$
T(V) \vDash F\left(T\left(v_{1}\right), \cdots, T\left(v_{m}\right)\right) \quad \text { iff } \quad V \vDash F\left(T\left(v_{1}\right), \cdots, T\left(v_{m}\right)\right) .
$$

Repeated use of the identity now produces a formula $G$ in $L n(S B K)$ and

$$
V \vDash F\left(v_{1}, \cdots, v_{m}\right) \text { iff } \operatorname{Det}(V) \vDash G\left(v_{1}, \cdots, v_{m}, c_{1}, \cdots, c_{n}\right) \text {. }
$$

Dropping the assumption on $\left[c_{1} \cdots c_{n}\right]$, we find that, for all $c_{1}, \cdots, c_{n}$,

$$
\operatorname{Det}(V) \vDash\left[c_{1} \cdots c_{n}\right] \neq 1 \vee G\left(v_{1}, \cdots, v_{m}, c_{1}, \cdots, c_{n}\right) \text {. }
$$

Therefore,

$$
\operatorname{Det}(V) \vDash \forall c_{1} \cdots \forall c_{n}\left(\left[c_{1} \cdots c_{n}\right] \neq 1 \vee G\left(v_{1}, \cdots, c_{n}\right)\right)
$$

and we see that

$$
V \vDash F\left(v_{1}, \cdots, v_{m}\right) \rightarrow \operatorname{Det}\left(\forall c_{1} \cdots \forall c_{n}\left(\left[c_{1} \cdots c_{n}\right] \neq 1 \vee G\right)\right) .
$$

Part II. Using this definition of $G$ we must verify that

$$
\operatorname{Det}\left(\forall c_{1} \cdots \forall c_{n}\left(\left[c_{1} \cdots c_{n}\right] \neq 1 \vee G\right)\right) \rightarrow F .
$$

Assume that $V \vDash \operatorname{Det}\left(G^{\prime}\right)$ and take the unit basis $b_{1}, \cdots, b_{n}$ for $V$. Substituting these for the $c_{i}$,

$$
\operatorname{Det}(V) \vDash\left[b_{1} \cdots b_{n}\right] \neq 1 \vee G\left(v_{1}, \cdots, v_{m}, b_{1}, \cdots, b_{n}\right) .
$$

However, $\operatorname{Det}(V) \vDash\left[b_{1} \cdots b_{n}\right]=1$ and $\operatorname{det}\left[b_{1} \cdots b_{i-1} z b_{i+1} \cdots b_{n}\right]=e^{i}(z)$ so

$$
\operatorname{Det}(V) \vDash G\left(v_{1}, \cdots, b_{n}\right) \text { and } V^{\prime} F F\left(v_{1}, \cdots, v_{m}\right) \text {. }
$$


We conclude that $\left[c_{1} \cdots c_{n}\right] \neq 1 \vee G\left(v_{1}, \cdots, c_{n}\right)$ is the required formula.

Part III. Assuming that $F \leftrightarrow \operatorname{Det}\left(\forall c_{1} \cdots \forall c_{n} G\right)$ for some open $G$ in $L n(S B K)$, we will verify that $F$ is invariant for $\operatorname{Sl}(n K)$ by checking this for $\operatorname{Det}\left(\forall c_{1} \ldots \forall c_{n} G\right)$.

Take a model $V$ and a morphism $T$ with domain $V$. Working within the field $S$ common to $V$ and $T(V)$, we have $\operatorname{det}[T]=1$ and

$$
\operatorname{det}\left[T\left(y_{1}\right) \cdots T\left(y_{n}\right)\right]=\operatorname{det}\left([T] \times\left[y_{1} \cdots y_{n}\right]\right)=\operatorname{det}\left[y_{1} \cdots y_{n}\right] .
$$

Substituting into $G$ we find

$$
\begin{aligned}
& V \vDash \operatorname{Det}\left(G\left(v_{1}, \ldots, v_{m}, c_{1}, \cdots, c_{n}\right)\right. \text { iff } \\
& T(V) \models \operatorname{Det}\left(G\left(T\left(v_{1}\right), \cdots, T\left(v_{m}\right), T\left(c_{1}\right), \ldots, T\left(c_{n}\right)\right)\right) .
\end{aligned}
$$

However, $T$ is surjective, so, as $c_{1}, \cdots, c_{n}$ cover $v, T\left(c_{1}\right), \cdots, T\left(c_{n}\right)$ cover $T(V)$. Thus,

$$
\begin{aligned}
& \operatorname{Det}(V) \vDash \forall c_{1} \cdots \forall c_{n} G\left(v_{1}, \cdots, c_{1}, \cdots, c_{n}\right. \text { iff } \\
& \operatorname{Det}(T(V)) \models \forall c_{1} \cdots \forall c_{n} G\left(T\left(v_{1}\right), \cdots, T\left(v_{m}\right), c_{1}, \cdots, c_{n}\right) .
\end{aligned}
$$

Therefore, $F$ is invariant for $S l(n K)$.

Before proceeding to invariance for $G l(n K)$. we need a lemma about open formulas in the language of integral domains which plays a role analogous to Lagrange's theorem for roots of polynomial equations. This lemma will just be stated here, as it has been presented els ewhere, together with some applications [12, 3.1].

Lemma 2.2. If $F$ is an open formula of the theory of integral domains and $K$ is an infinite field, then $K \vDash F\left(a_{1}, \cdots, a_{m}, t\right)$ for infinitely many $t$ iff $K \vDash F^{\prime}\left(a_{1}, \cdots, a_{m}\right)$ where $F^{\prime}$ comes from $F$ by replacing each equation, $\left(\Sigma p_{i} t^{i}\right)=0$, by the formula $\Pi\left(p_{i}=0\right)$. Here $\Pi$ stands for the conjunction of the formulas.

This lemma is an extension of the usual technique for homogenizing equations by taking their homogeneous parts. We can now give necessary and sufficient conditions for invariance for $G l(n K)$.

Theorem 2.3. An open formula $F$ in $L n(S A K)$ is invariant for $G l(n K)$ iff there is a quantifier-free, homogeneous formula $G$ in $L n(S B K)$, with the same free variables plus $n$ new variables $c_{1}, \cdots, c_{n}$, such that $F \leftrightarrow \operatorname{Det}\left(\forall c_{1} \cdots \forall c_{n} G\right)$ in the the ory of $V n K$.

Proof. Part I. Assume $F$ is invariant for $G l(n K)$. Take any model $V$ in $V n K$ and extend it to a model $\inf (V)$ with an infinite field $\inf (S)$. Since $F$ is open, for any $v_{1}, \cdots, v_{m}$ in $V$ 


$$
V \vDash F\left(v_{1}, \cdots, v_{m}\right) \text { iff } \inf (V) \vDash F\left(v_{1}, \cdots, v_{m}\right) \text {. }
$$

Take the transformation in $G l(n K)$ with matrix $s \cdot I_{n}, s \not 0$ in inf $(s)$ means $\operatorname{det}[T]=s^{n}$. Therefore,

$$
\inf (V) \vDash F\left(v_{1}, \cdots, v_{n}\right) \quad \text { iff } \quad T(\inf (V)) \vDash F\left(T\left(v_{1}\right), \cdots, T\left(v_{m}\right)\right) .
$$

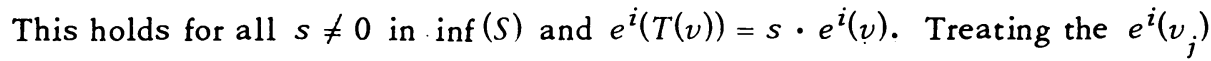
as elements of the field, we find that this is a formula which holds for infinitely many $s$. Applying Lemma 2.2, we get $\inf (V) \vDash F^{\prime}\left(v_{1}, \cdots, v_{m}\right)$ where $F^{\prime}$ comes from separating the coefficients of $s^{i}$ in $F$. This makes $F^{\prime}$ homogeneous in the $e^{i}\left(v_{j}\right)$ and since $F^{\prime}$ is also an open formula

$$
V \models F\left(v_{1}, \cdots, v_{m}\right) \quad \text { iff } \quad V^{\prime} F F^{\prime}\left(v_{1}, \cdots, v_{m}\right) .
$$

We can now apply to $F^{\prime}$ the transformation $T$ which comes from the vectors $c_{1}, \cdots, c_{n}$, such that $\operatorname{det}\left[c_{1} \cdots c_{n}\right] \neq 0$, as in the proof of Theorem 2.1. From this we derive that if $V \vDash F\left(v_{1}, \cdots, v_{m}\right)$ then, for all $c_{1}, \cdots, c_{n}$ in $V$,

$$
\operatorname{Det}(V) \vDash\left[c_{1} \cdots c_{n}\right]=0 \vee G^{\prime}\left(v_{1}, \cdots, v_{m}, c_{1}, \ldots, c_{n}\right) \text {. }
$$

Thus,

$$
\begin{gathered}
V \vDash F\left(v_{1}, \cdots, v_{m}\right) \rightarrow \operatorname{Det}\left(\forall c_{1} \ldots \forall c_{n}\left(\left[c_{1} \cdots c_{n}\right]=0 \vee G^{\prime}\left(v_{1}, \cdots, c_{n}\right)\right)\right) . \\
\quad \text { If } \operatorname{Det}(V) \vDash \forall c_{1} \cdots \forall c_{n}\left(\left[c_{1} \cdots c_{n}\right]=0 \vee G^{\prime}\left(v_{1}, \cdots, c_{n}\right)\right) \text { then, taking }
\end{gathered}
$$
the unit basis for $V, b_{1}, \cdots, b_{n}$, we have $\operatorname{Det}(V) \vDash\left[b_{1} \cdots b_{n}\right] \neq 0$ and therefore, after substitution $\operatorname{Det}(V) \vDash G^{\prime}\left(v_{1}, \cdots, v_{m}, b_{1}, \cdots, b_{n}\right)$. Since $\operatorname{det}\left[b_{1} \cdots b_{i-1} v b_{i+1} \cdots b_{n}\right]=e^{i}(v)$, we conclude that $V \vDash F^{\prime}\left(v_{1}, \cdots, v_{m}\right)$ and therefore $V \vDash F\left(v_{1}, \cdots, v_{m}\right)$.

This formula is homogeneous in the brackets because the homogeneity of $F^{\prime}$ produced the necessary homogeneity in $G^{\prime}$.

Part II. Assume there is a homogeneous open $G$ such that $F \leftrightarrow$ $\operatorname{Det}\left(\left(\forall c_{1}, \cdots, c_{n}\right) G\right)$. Take any morphism in $G l(n K), T$, with domain $V$. It suffices to prove that $\forall c_{1} \cdots \forall c_{n} G$ is invariant under $T$.

In the field $S$ common to $V$ and $T(V)$ we find that $\operatorname{det}\left[T\left(y_{1}\right) \cdots T\left(y_{n}\right)\right]=$ $\operatorname{det}\left([T] \times\left[y_{1} \cdots y_{n}\right]\right)=\operatorname{det}[T] \cdot \operatorname{det}\left[y_{1} \cdots y_{n}\right]$. Since $G$ is homogeneous in the brackets and $\operatorname{det}[T] \neq 0$, we have

$$
\operatorname{Det}(V) \vDash G\left(v_{1}, \cdots, c_{n}\right) \quad \text { iff } \quad \operatorname{Det}(T(V)) \vDash G\left(T\left(v_{1}\right), \ldots, T\left(c_{n}\right)\right) \text {. }
$$

Also, while $c_{i}$ ranges over $V, T\left(c_{i}\right)$ covers $T(V)$ so

$$
\begin{aligned}
& \operatorname{Det}(V) \vDash \forall c_{1} \cdots \forall c_{n} G\left(v_{1}, \cdots, c_{n}\right) \text { iff }
\end{aligned}
$$

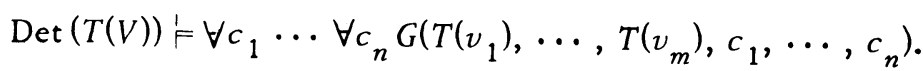


We conclude that $V \vDash F\left(v_{1}, \cdots, v_{m}\right)$ iff $T(V) \vDash F\left(T\left(v_{1}\right), \cdots, T\left(v_{m}\right)\right)$.

For the category $\mathrm{Pa}(n \mathrm{~K})$ of all collineations of projective geometry we have a necessary and sufficient condition for invariance for only a small class of fields $K$, the prime fields and $\varnothing$. We approach it this way.

Definition 2.1. A field $K$ is fixed iff any automorphism of a field $K^{\prime}$ extending $K$, is the identity map on $K$.

The obvious examples of fixed fields are the prime fields and the empty field $\varnothing$. From results of universal a lgebra we can prove that these are the only fixed fields [5, p. 184]. Fortunately, these do include the principal cases of geometric importance.

Theorem 2.4. An open formula $F$ in $L n(S A K)$, involving only the constants 0 and 1 , is invariant for $\mathrm{Pa}(n \mathrm{~K})$ iff there is an open, bomogeneous formula $G$ in $L n(S B K)$, involving only the constants 0 and 1 , with the same free variables plus $c_{1}, \cdots, c_{n}$, such that $F \leftrightarrow \operatorname{Det}\left(\forall c_{1} \cdots \forall c_{n} G\right)$ in the theory of $V n K$.

Proof. Part I. Since $F$ is invariant for $P a(n K)$ it is invariant for $G l(n K)$ and by Theorem 2.3 there is the required $G$ with the same constants.

Part II. Assume $F \leftrightarrow \operatorname{Det}\left(\forall c_{1} \ldots \forall c_{n} G\right)$ with $G$ as in the theorem.

By Theorem 2.3, $G$ is invariant for all morphisms in $G l(n K)$. It remains to check that $G$ is invariant under morphisms induced by an isomorphism, $a$, of the underlying field $S$ on a model $V$. Consider any atomic formula of $\operatorname{Det}(G)$, which is essentially of the form $\Sigma f_{j} \cdot p_{j}=0$ where the $p_{j}$ are monomials in the $e^{i}(v)$ and $e^{i}(c)$. Now in the field: $e^{i}(T(v))=a\left(e^{i}(v)\right)$ and $a(f)=f$ since $f$ is in the prime field of the model. Thus we have

$$
\sum f_{j} \cdot \prod e^{i}(T(y))=\sum f_{j} \cdot \prod a\left(e^{i}(y)\right)=\sum a\left(f_{j}\right) \cdot \prod a\left(e^{i}(y)\right)=a\left(\sum f_{j} \cdot \prod e^{i}(y)\right)
$$

and $a[0]=0$. Therefore $\operatorname{Det}(V) \vDash G\left(v_{1}, \cdots, c_{n}\right)$ for all $c_{i}$ in $V$ iff $\operatorname{Det}(T(V)) \vDash G\left(T\left(v_{1}\right), \cdots, T\left(c_{n}\right)\right)$ for all $T\left(c_{i}\right)$ in $T(V)$. Since $T$ is surjective this concludes the proof.

The existence of a formula $G$ of this form is always sufficient for the invariance of $F$, no matter what coefficients occur in $F$. However, it remains a conjecture that this is a necessary condition, even for open formulas. For a single equation and a field of any characteristic we have verified the necessity, as well as for open formulas in a single variable over any field. In general it remains unknown.

3. Invariant formulas with quantifiers. In all categories we have characterizations for general formulas which directly extend the results of $\$ 2$. 
Theorem 3.1. A formula $F$ in $L n(S A K)$ is invariant for $S l(n K)$ iff there is a formula $G$ in $L n(S B K)$, with the same free variables and the same bound variables plus $c_{1}, \cdots, c_{n}$, such that: $F \leftrightarrow \operatorname{Det}(G)$ in the theory of $V n K$.

Proof. Part I. Assume that $F$ is invariant for $S l(n K)$. Take a model $V$ and consider the Skolem form of the formula in $V, F^{s}\left(v_{1}, \cdots, v_{m}, y_{1}, \cdots, y_{p}, s_{1}, \cdots, s_{q}\right)$ which results from putting $F$ in prenex normal form, with all its quantifiers in the front, replacing the universal quantifiers by new variables $y_{1}, \cdots, y_{p}$ and replacing the existential quantifiers by Skolem functions in the model $V: s_{1}, \ldots$, $s_{q}$, each function depending on the variables $v_{1}, \cdots, v_{m}$ and those $y_{i}$ which lie outside of the existential quantifier in the prefix of $F$. The principal property of the Skolem form of a formula is this

$$
V \vDash F\left(a_{1}, \cdots, a_{m}\right) \quad \text { iff } \quad V=F^{s}\left(a_{1}, \cdots, a_{m}, b_{1}, \cdots, b_{p}, s_{1}, \cdots, s_{q}\right)
$$

for all $b_{1}, \cdots, b_{p}$ in $V$.

We can now operate on $F^{s}$ much as we did on the open formulas in Theorem 2.1.

$$
\begin{aligned}
& V^{\prime} F F\left(a_{1}, \cdots, a_{m}\right) \quad \text { iff } \quad T(V)_{F}^{\prime}=F\left(T\left(a_{1}\right), \ldots, T\left(a_{m}\right)\right) \\
& \text { iff } \quad T(V) \vDash F^{s}\left(T\left(a_{1}\right), \cdots, T\left(a_{m}\right), b_{1}, \cdots, b_{p}, s_{1}, \cdots, s_{q}\right) \\
& \text { iff } \quad T(V) \vDash F^{s}\left(T\left(a_{1}\right), \cdots, T\left(a_{m}\right), T\left(T^{-1}\left(b_{1}\right)\right), \cdots\right. \text {, } \\
& \left.T\left(T^{-1}\left(b_{p}\right)\right), T\left(T^{-1}\left(s_{1}\right)\right), \ldots, T\left(T^{-1}\left(s_{q}\right)\right)\right) .
\end{aligned}
$$

Since $T$ is a bijection and $T^{-1}(x)$ is a point in $T(V), T^{-1}\left(s_{j}\right)$ can be considered as new Skolem functions in $T(V)$. Thus we have

$$
T(V) \vDash F^{s}\left(T\left(a_{1}\right), \ldots, T\left(a_{m}\right), T\left(d_{1}\right), \ldots, T\left(d_{p}\right), T\left(s_{1}^{\prime}\right), \ldots, T\left(s_{q}^{\prime}\right)\right)
$$

for all $d_{1}, \cdots, d_{n}$ in $V$.

Using the same trick as in Theorem 2.1 , we introduce the new $c_{1}, \cdots, c_{n}$ corresponding to $T$ and find that

$$
\operatorname{Det}(V) \vDash G^{s}\left(a_{1}, \cdots, a_{m}, d_{1}, \cdots, d_{p}, c_{1}, \cdots, c_{n}, s_{1}^{\prime}, \cdots, s_{q}^{\prime}\right)
$$

for all $d_{i}$ in $\operatorname{Det}(V)$ and all $c_{j}$ such that $\left[c_{1} \cdots c_{n}\right]=1$. The $s_{k}^{\prime}$ depend also on the new variables $c_{1}, \cdots, c_{n}$. Thus, $\operatorname{Det}(V) \vDash\left[c_{1} \cdots c_{n}\right] \neq 1 \mathrm{~V}$ $G\left(a_{1}, \cdots, a_{m}, c_{1}, \cdots, c_{n}\right)$, for all $c_{j}$. We conclude that

$$
V \vDash F\left(a_{1}, \cdots, a_{m}\right) \rightarrow \operatorname{Det}\left(\forall c_{1} \ldots \forall c_{n}\left(\left[c_{1} \cdots c_{n}\right] \neq 1 \vee G\left(a_{1}, \cdots, c_{n}\right)\right)\right) .
$$

The construction of $G$ assures us that $G$ has the correct free and bound variables.

That $V \vDash \operatorname{Det}\left(\forall c_{1} \cdots \forall c_{n}\left(\left[c_{1} \cdots c_{n}\right] \neq 1 \vee G\right)\right) \rightarrow F$ follows from the usual substitution. 
Part II. Assume $F \leftrightarrow \operatorname{Det}(G)$. Take any model $V$ and morphism $T$ in $S l(n K)$, with domain $V$. Using the identity in the field $S$ common to $V$ and $T(V)$, $\operatorname{det}\left[T\left(y_{1}\right) \cdots T\left(y_{n}\right)\right]=\operatorname{det}\left[y_{1} \cdots y_{n}\right]$, we have

$$
\begin{aligned}
& \operatorname{Det}(V) \vDash G\left(a_{1}, \ldots, a_{m}\right) \text { iff } \operatorname{Det}(V) G^{s}\left(a_{1}, \ldots, b_{i}, \ldots, s_{q}\right) \\
& \text { iff } \operatorname{Det}(T(V)) \vDash G^{s}\left(T\left(a_{1}\right), \ldots, T\left(b_{i}\right), \ldots, T\left(s_{q}\right)\right) \\
& \text { iff } \operatorname{Det}(V) \vDash G\left(T\left(a_{1}\right), \ldots, T\left(a_{m}\right)\right),
\end{aligned}
$$

since $T$ is surjective and $T\left(s_{j}\right)$ is a new Skolem function.

Thus $F$ is invariant for $\operatorname{sl}(n K)$.

Theorem 3.2. A formula $F$ in $L n(S A K)$ is invariant for $G l(n K)$ iff there is a bomogeneous formula $G$ in $L n(S B K)$, with the same free variables and the same bound variables plus $c_{1}, \cdots, c_{n}$, such that $F \leftrightarrow \operatorname{Det}(G)$ in the theory of $V n K$.

Proof. We modify the proof of Theorem 3.1 by applying the transformation $T$ with matrix $\left[c_{1} \cdots c_{n}\right]^{-1}$. That is, $T_{i j}=$ (coefficient of $c_{j i}$ in the expansion of $\operatorname{det}\left[c_{1} \cdots c_{n}\right]$ by the $i$ th column $) \div \operatorname{det}\left[c_{1} \cdots c_{n}\right]$, provided $\operatorname{det}\left[c_{1} \cdots c_{n}\right] \neq 0$.

Now we find $e^{i}(T(v))=\Sigma T_{i j} \cdot e^{j}(v)=\operatorname{det}\left[c_{1} \cdots v \cdots c_{n}\right] \div \operatorname{det}\left[c_{1} \cdots c_{n}\right]$. After repeated use of these identities and multiplication by an appropriate power of $\operatorname{det}\left[c_{1} \cdots c_{n}\right]$ to clear fractions, we find $F$ has beer transformed into a homogeneous formula $G$. As before we can verify that $F \leftrightarrow \operatorname{det}\left(\forall c_{1} \cdots \forall c_{n}\left(\left[c_{1} \cdots c_{n}\right]=0 \vee G\right)\right)$ which gives us the required homogeneous $G^{\prime}$.

The procedure for checking that such $G$ are all invariant for $G l(n K)$ is identical to that for Theorem 3.1 employing Skolem forms and using the identity for homogeneity from the proof of Theorem 2.3.

Corollary 3.3. If $F$ is a formula with only constants 0 and 1 in $\operatorname{Ln}(S A K)$ then $F$ is invariant for $\mathrm{Pa}(n K)$ iff there is a bomogeneous formula $G$ in $L n(S B K)$, with the same constants, and free variables, sucb that $F \leftrightarrow \operatorname{Det}(G)$ in the theory of $V n K$.

Proof. Simply combine the proof of Theorem 2.4 with Theorem 3.2.

The procedure used in Theorem 3.2 could have been used on the open formulas in Theorem 2.3 but it obscures the information on the homogeneous parts of $F$ which we obtained in that case and thus provides less useful information on the structure of $G$. In both cases the construction of $G$ is constructive and practicable.

4. Covariant and contravariant vectors. For reasons related to geometric duality and to tensors, significant portions of the classical invariant theory were concerned with languages which included coordinates of "contravariant 
vectors"', $A, B, \cdots$, as well as covariant vectors, $v, x, \cdots$. Geometrically such vectors represent hyperplanes in the projective geometry. In this section we will indicate how the preceding discussion can be modified to apply equally well to this broader context.

Definition 4.1. The language $L n(C A K)$ for analytic geometry with covariant and contravariant vectors is a modified three-sorted language of type

$$
\tau=\left\{V, H, S ;=; e^{1}, \cdots, e^{n}, e_{1}, \cdots, e_{n},+, \cdot,-; 0,1,\left\langle c_{f}\right\rangle_{f \in K}\right\}
$$

and signature

$$
\begin{aligned}
& \sigma:=\text { is a relation on terms of sort } S \\
& e^{1}, \cdots, e^{n} \text { are operations } V \rightarrow S, \\
& e_{1}, \cdots, e_{n} \text { are operations } H \rightarrow S, \\
& \quad+\text { and }- \text { are operations } S \times S \rightarrow S, \\
& \quad-\text { is an operation } S \rightarrow S, \\
& 0,1 \text { and } c_{f}, f \in K, \text { are constants of sort } S, \\
& \\
& \text { and only variables of sorts } V \text { and } H \text { occur in the language. }
\end{aligned}
$$

Definition 4.2. A standard model of $L n(C A K)$ is a model

$$
C=\left\{V, H, S ;=; e^{1}, \cdots, e^{n}, e_{1}, \cdots, e_{n},+, \cdot,-; 0,1,\langle b(f)\rangle_{f \in K}\right\}
$$

where $S$ is a field, $V$ and $H$ are vector spaces of dimension $n$ over $S$, and

$=$ is the equality relation on $S$,

$e^{1}, \cdots, e^{n}$ are the projections from $V$ into $S$,

$e_{1}, \cdots, e_{n}$ are the projections from $H$ into $S$,

,$+ \cdot$ and - are the usual operations on $S$,

0 and 1 are the zero and unit of $S$,

and $b$ is a field injection of $K$ into $S$.

The collection of all the standard models is denoted $C n K$.

When we come to set up the transformations on the models we find that, in order to correspond to a projectivity or change of coordinates, a transformation with matrix $[T]$ which takes $v$ in $V$ into $[T] \times[v]$ for $[v]$ a column vector must act contagradiently on $H$ and take $B$ in $H$ into $[B] \times[T]^{-1}$ for $[B]$ a row vector. This is necessary to preserve as simple a property as the point $v$ is in the hyperspace $B$. For this reason the elements of $H$ are called contravariant vectors, while the elements of $V$ are called covariant vectors.

We can now set up the series of categories by introducing the appropriate morphisms on the models $C n K$.

Definition 4.2. $S l(n C K)$ is the category with objects $C n K$ and the morphisms for a model $C$ are all transformations induced by matrices [T] over the field $S$ with $\operatorname{det}[T]=1$, where $T$ acts cogradiently on $V$ and contragradiently on $H$. 
Definition 4.3. $G l(n C K)$ is the category with objects $C n K$ and morphisms for a model $C$ given by matrices $[T]$ over $S$, $\operatorname{det}[T] \neq 0$, where $T$ acts cogradiently on $V$ and contragradiently on $H$.

Definition 4.4. $P a(n C K)$ is the category with objects $C n K$ and morphisms $T$ induced by isomorphisms of the fields, $a$, such that $T\left(x_{1}, \cdots, x_{n}\right)=\left(a\left(x_{1}\right), \cdots, a\left(x_{n}\right)\right)$ and $T\left(A^{1}, \ldots, A^{n}\right)=\left(a\left(A^{1}\right), \ldots, a\left(A^{n}\right)\right)$, as well as all morphisms of $G l(n C K)$ and compositions of such morphisms.

The definition of a formula being invariant for any of these categories gives, in each case, a notion which extends the definition for formulas with only covariant vectors.

Once more, from the classical theory we have a hunch about the syntactic form of invariant formulas.

Definition 4.5. The language $L n(S C K)$, a language for covariant and contravariant vectors, is a modified three-sorted language of type

$$
\tau=\left\{V, H, S ;=;[],[]^{\prime},(),+, \cdot,-; 0,1,\left\langle c_{f}\right\rangle_{f \in K}\right\}
$$

and signature

$\sigma:=$ is a relation on terms of type $S$

[] is an operation $V^{n} \rightarrow S$,

[ ] $]^{\prime}$ is an operation $H^{n} \rightarrow S$,

( ) is an operation $H \times V \rightarrow S$,

+ and $\cdot$ are operations $S \times S \rightarrow S$,

- is an operation $S \times S \rightarrow S$,

0,1 and $c_{f}, f \in K$, are constants of sort $S$,

and only variables of sorts $V$ and $H$ occur in the language.

Definition 4.6. A standard model of $L n(S C K)$ is a model

$$
D=\left\{V, H, S ;=; \operatorname{det}, \operatorname{det}^{\prime},(),+, \cdot,-; 0,1,\langle b(f)\rangle_{f \in K^{\prime}}\right\}
$$

where $V$ and $H$ are vector spaces of dimension $n$ over the field $S$ and

$=$ is the equality relation on $S$,

det is the operator $\operatorname{det}\left[v_{1} \cdots v_{n}\right]$, with the vectors written as columns of the matrix,

$\operatorname{det}^{\prime}$ is the operator $\operatorname{det}\left[A_{1} \cdots A_{n}\right]$, with the vectors written as rows of the matrix,

( ) is the operation $[A] \times[v]$, where $A$ is written as a row vector, $v$ as a column vector, and the resulting $1 \times 1$ matrix is identified with the element of the field; it is the dot product of the two vectors,

,$+ \cdot$, and - are the usual operations on the field $S$, 
0 and 1 are the zero and identity of $S$,

$b$ is an embedding of $K$ into $S$.

The collection of all standard models is denoted $D n K$.

As a convention we will denote constants and variables of sort $V$ by small letters, usually from the end of the alphabet: $v, v_{1}, \cdots, x, \cdots$ etc., and constants and variables of sort $H$ by capital letters, usually from the beginning of the alphabet: $A, A_{1}, \cdots, B, \cdots$ etc. Since it will be clear from the sort of terms inside whether we are in $V$ or $H$, we drop the distinctions between [ ] and [ ]', as well as det and det', by omitting all primes.

We extend the operator Det to be from $\operatorname{Ln}(S C K)$ to $\operatorname{Ln}(C A K)$ by defining the operation on basic terms as follows

$$
\begin{aligned}
\operatorname{Det}\left(\left[y_{1} \cdots y_{n}\right]\right. & =\operatorname{det}\left(e^{i}\left(y_{j}\right)\right), \\
\operatorname{Det}\left(\left[A_{1} \cdots A_{n}\right)\right] & =\operatorname{det}\left(e_{i}\left(A_{j}\right)\right), \\
\operatorname{Det}((A y)) & =\sum e_{i}(A) \cdot e^{i}(y)
\end{aligned}
$$

and extending linearly to all terms and formulas.

The corresponding operator Det from $C n K$ to $D n K$ is similarly defined.

To extend the theorems of the earlier sections all that remains is to define homogeneous formulas appropriately for $L n(S C K)$. Because of the differing action of the morphisms on covariant and contravariant vectors, the total degree of a polynomial in all its variables is the total degree in the covariant variables, less the total degree in the contravariant variables, except for the zero polynomial which has, as always, total degree $-\infty$. For example a term like $(A y)$ has total degree 0 and a term like $1+(A y)+\left[A_{1} \cdots A_{n}\right] \cdot\left[y_{1} \cdots y_{n}\right]$ is homogeneous of degree 0 .

With this definition of homogeneous terms extended to define homogeneous equations and formulas, all of the theorems of $\$ \$ 2$ and 3 apply with $\operatorname{Ln}(C A K)$ replacing $L n(S A K), L n(S C K)$ replacing $L n(S B K)$ and $C n K$ replacing $V n K$.

We will give two examples of this translation and illustrate the modifications required in the proofs. The first example is a direct translation of Theorem 2.1.

Theorem 4.1. An open formula $F$ in $L n(C A K)$ is invariant for $\operatorname{sl}(n C K)$ iff there is an open formula $G$ in $L n(S C K)$, with the same free variables plus perbaps $n$ new covariant variables $c_{1}, \cdots, c_{n}$, such that $F \leftrightarrow \operatorname{Det}\left(\forall c_{1} \cdots \forall c_{n} G\right)$ in all models of $\mathrm{CnK}$.

Proof. Part I. Assume $F$ is invariant and take a model $C$ in $C n K$ and covariant vectors $c_{1}, \cdots, c_{n}$ in $C$. Consider the transformation $T$ with matrix $\left[T_{i j}\right]$, where $T_{i j}$ is the cofactor of $e^{i}\left(c_{j}\right)$ in $\left[c_{1} \cdots c_{n}\right]$. If $\operatorname{det}\left[c_{1} \cdots c_{n}\right]=1$ then $\operatorname{det}[T]=1$ and $T$ is in $\operatorname{Sl}(n K)$. In this case we have 


$$
\begin{aligned}
& C \vDash F\left(v_{1}, \cdots, v_{m}, A_{1}, \cdots, A_{p}\right) \text { iff } \\
& T(C) \models F\left(T\left(v_{1}\right), \cdots, T\left(v_{m}\right), T\left(A_{1}\right), \cdots, T\left(A_{p}\right)\right) .
\end{aligned}
$$

In the field $S$ of $C$ and $T(C)$, since $\left[c_{1} \cdots c_{n}\right]$ is the matrix of $T^{-1}$, we have

$$
\begin{aligned}
& e^{i}(T(y))=\operatorname{Det}\left[c_{1} \cdots c_{i-1} y c_{i+1} \cdots c_{n}\right], \\
& e_{i}(T(A))=\sum e^{j}\left(c_{i}\right) \cdot e_{j}(A)=\operatorname{Det}\left(A c_{i}\right) .
\end{aligned}
$$

Therefore, this procedure carries $F$ into a formula $G^{\prime}$ in $\operatorname{Ln}(S C K)$ such that

$$
\begin{aligned}
C & \models F\left(v_{1}, \cdots, v_{m}, A_{1}, \cdots, A_{p}\right) \\
& \rightarrow \operatorname{Det}\left(\forall c_{1} \cdots \forall c_{n}\left(\left[c_{1} \cdots a_{n}\right] \neq 1 \vee G^{\prime}\left(v_{1}, \cdots, A_{p}\right)\right)\right) .
\end{aligned}
$$

Part II. With $G^{\prime}$ defined as above we can substitute a unit basis $b_{1}, \ldots$, $b_{n}$ for $c_{1}, \cdots, c_{n}$ and find that

$$
\begin{aligned}
\operatorname{Det}\left[b_{1} \cdots b_{i-1} y \dot{b}_{i+1} \cdots b_{n}\right] & =e^{i(y)} \\
\operatorname{Det}\left(A b_{i}\right) & =e_{i}(A), \\
\operatorname{Det}\left[b_{1} \cdots b_{n}\right] & =1 .
\end{aligned}
$$

Thus, as always, $C \vDash \operatorname{Det}\left(\forall c_{1} \cdots \forall c_{n}\left(\left[c_{1} \cdots c_{n}\right] \neq 1 \vee G^{\prime}\left(v_{1}, \cdots, A_{p}\right)\right)\right) \rightarrow$ $F\left(v_{1}, \cdots, A_{p}\right)$.

Part III. We will simply note the identities required

$$
\begin{aligned}
\operatorname{Det}\left[T\left(y_{1}\right) \cdots T\left(y_{n}\right)\right] & =\operatorname{det}[T] \cdot \operatorname{det}\left[y_{1} \cdots y_{n}\right]=\operatorname{Det}\left[y_{1} \cdots y_{n}\right], \\
\operatorname{Det}(T(A y)) & =[A] \times\left[T^{-1}\right] \times[T] \times[y]=[A] \times[y]=\operatorname{Det}(A y), \\
\operatorname{Det}\left[T\left(A_{1}\right) \cdots T\left(A_{n}\right)\right] & =\operatorname{det}\left[A_{1} \cdots A_{n}\right] \cdot \operatorname{det}\left[T^{-1}\right]=\operatorname{Det}\left[A_{1} \cdots A_{n}\right] .
\end{aligned}
$$

The rest of the proof follows Theorem 2.1.

The second example will illustrate the role of our definition of homogeneity.

Theorem 4.2. An open formula $F$ in $L n(C A K)$ is invariant for $G l(n K)$ iff there is an open, homogeneous formula $G$ in $L n(S C K)$, with the same free variables plus perbaps $n$ new covariant variables $c_{1}, \cdots, c_{n}$, sucb that $F \leftrightarrow \operatorname{Det}\left(\forall c_{1} \ldots \forall c_{n} G\right)$ in all models in $C n K$.

Proof. Part I. Assume that $F$ is invariant for $G l(n K)$. Take any infinite model $C$ in $C n K$ and consider the transformation $T$ with matrix $s I_{n}, s \neq 0$. Then

$$
\begin{aligned}
& C \models F\left(v_{1}, \cdots, v_{m}, A_{1}, \cdots, A_{p}\right) \quad \text { iff } \\
& T(C) \models F\left(T\left(v_{1}\right), \cdots, T\left(v_{m}\right), T\left(A_{1}\right), \cdots, T\left(A_{p}\right)\right) .
\end{aligned}
$$

In the infinite field $S$ of $C$ and $T(C)$ we find $e^{i}(T(y))=s \cdot e^{i}(y)$ and $e_{j}(T(A))$ $=s^{-1} \cdot e_{j}(A)$. After multiplying by some power of $s$ to clear lingering $s^{-1}$, 
we obtain a formula $F^{\prime \prime}$ satisfied by an infinite set of $s$ in the field $S$. Since the coefficients of $s^{r}$ in this formula are homogeneous polynomials in our sense, after application of Lemma 2.2, we have an open, homogeneous formula $F^{\prime}$.

The rest of the proof follows that of Theorem 2.3 and the definition of homogeneity is correct to verify that all such homogeneous $G$ must be invariant for $G l(n K)$.

The reader can now translate and prove any other of the theorems of the preceding sections. The sharp eyed reader may have noticed that, for the creation of $G$ corresponding to an invariant formula $F$, we never needed the existence of the operation $\left[A_{1} \cdots A_{n}\right]$ in the language $L n(S C K)$. We have chosen to include this operation both to emphasize the essential duality of the contravariant and covariant variables, particularly in later work when we will be calculating and doing proofs in these formulas, and because, historically, it has always been present in the work on invariants, for reasons related to the First Fundamental Theorem of Invariant Theory.

5. Klein's Erlanger program and other categories. It is now clear that the basis of Klein's program can be restated so that a geometry is given by means of a category of models. The geometric properties are those properties expressed by formulas invariant for the category. The geometric theorems are those sentences, built up from the invariant formulas, which are true in all the models in the category.

Driven by a strong intution, geometers endure more severe problems with algebraic languages than other mathematicians. They begin with a category of models, say vector spaces, which is very meagre in morphisms, though rich in algebraic structure. Then they enrich the category with the necessary geometric morphisms, for example the collineations, and struggle to separate out of the "bad" models that essential structure and language which is invariant under the geometric transformations. The richer the morphisms, the smaller the collection of formulas which remains and the more of the tangled algebraic structure which must be forgotten.

The classical examples of geometry: euclidean, affine, and projective, were all stated in such a way that the categories chosen were groupoids. Then, for differential geometry, the categories were pseudogroups [9, pp. 31-41]. Even this restriction seems unnecessarily severe.

In our paper IV we will examine a category associated with combinatorial geometry which includes embeddings and is not even a pseudogroup. In our paper II we examine invariance for a more abstract category of first-order valuations. It remains true that the concept of invariance for a category is basic to any discussion of analytic geometry. 
6. Conclusion. We have not been totally successful in characterizing all formulas invariant under our fullest category, $\mathrm{Pa}(n \mathrm{~K})$. However, we have characterized the invariant formulas where $K$ is a fixed field, a much broader class of formulas and a fuller category than previously considered. In addition we have placed all invariant properties, for each of the categories examined, firmly inside the language of brackets and thus laid the foundation for considering all theorems of geometry as expressions in this language.

There may be an objection to the models chosen here to study projective properties - the zero vector does not represent a projective point and should not appear. It is perfectly feasible to create revised categories by removing the zero vector from each of the models. Since all the original morphisms are bijections which carry the zero vector to the zero vector, their restrictions are morphisms of the revised category. All of the operations in the languages $L n(S A K)$ and $L n(S B K)$ are well defined in these revised models. Even better, all of the theorems and proofs translate directly, with equivalence of the formulas $F$ and $\operatorname{Det}(G)$ in the theory of the revised models, while invariance of $F$ is stated in terms of the revised categories. This means that the same formulas are invariant and the same standard forms occur for invariant formulas, though the quantified formulas may represent different properties in the two kinds of categories.

We recall that the process of creating a formula in the brackets, equivalent to an invariant formula, is thoroughly constructive, as are the homogenizing techniques. This translation does not increase the complexity of formula as measured by the quantifier alternations, except for quantifier free formulas. In practice there is usually a simplification in the algebraic expressions as we move into the brackets.

The whole process of defining invariance can be applied to a single model and its endomorphisms. All the techniques apply to a single infinite model, to characterize the invariant formulas. The techniques of $\$ 3$ would apply to a single model of any cardinality.

Finally, the prolonged experience of analytic geometry and, in particular, the work in Grassman's calculus [4], shows that synthetic geometric properties. tend to translate as homogeneous formulas with coefficients 0 and 1 and are therefore within the class we have characterized.

\section{BIBLIOGRAPHY}

1. R. Baer, Linear algebra and projective geometry, Academic Press, New York, 1952. MR 14, 675.

2. J. A. Dieudonné and J. B. Carrell, Invariant theory, old and new, Advances in Math. 4 (1970), 1-80. MR $41 \# 186$. 
3. S. Feferman, Lectures on proof theory, Proc. Summer School in Logic (Leeds 1967), Springer, Berlin, 1968, pp. 1-107. MR 38 \#294.

4. H. G. Forder, The calculus of extension, Chelsea, New York, 1960. MR $22 \# 7009$.

5. B. Jónsson, Algebraic extensions of relational systems, Math. Scand. 11 (1962), 179-205. MR $27 \# 4777$.

6. F. Klein, Elementary mathematics from an advanced standpoint: Geometry, Dover, New York, 1939.

7. F. I. Mautner, An extension of Klein's Erlanger program: logic as invariant-theory, Amer. J. Math. 68 (1946), 345-384. MR 8, 3.

8. H. W. Turnbull, The the ory of determinants, matrices, and invariants, 3rd ed., Dover, New York, 1960. MR 24 \#A123.

9. O. Veblen and J. Whitehead, The foundations of differential geometry, Cambridge Univ. Press, Cambridge, 1932.

10. H. Weyl, The classical groups. Their invariants and representation, 2 nd ed., Princeton Univ. Press, Princeton, N. J., 1946.

11. W. Whiteley, Logic and invariant theory, Ph.D. Thesis, M. I. T., Cambridge, Mass., 1971.

12. - Homogeneous sets and homogeneous formulas, Rend. Mat. (to appear).

13. - Logic and invariant theory. II: Homogeneous coordinates (to appear).

14. N. White, The bracket ring and combinatorial geometry, Ph.D. Thesis, Harvard University, Cambridge, Mass., 1971. CANADA

DEPARTMENT OF MATHEMATICS, LAKEHEAD UNIVERSITY, THUNDER BAY, ONTARIO,

Current address: Department of Mathematics, Champlain Regional College, St. Lambert, Quebec, Canada 